\title{
Hörmander-type theorem for Itô processes and related backward SPDEs
}

\section{JINNIAO QIU}

Department of Mathematics and Statistics, University of Calgary, 2500 University Drive NW, Calgary, AB T2N 1N4, Canada. E-mail: qiujinn@gmail.com

A Hörmander-type theorem is established for Itô processes and related backward stochastic partial differential equations (BSPDEs). A short self-contained proof is also provided for the $L^{2}$-theory of linear, possibly degenerate BSPDEs, in which new gradient estimates are obtained.

Keywords: backward stochastic partial differential equation; Hörmander theorem; Itô process; non-Markov

\section{Introduction}

Let $\left(\Omega, \overline{\mathscr{F}},\left\{\overline{\mathscr{F}}_{t}\right\}_{t \geq 0}, \mathbb{P}\right)$ be a complete filtered probability space, on which two independent $d_{1}$ dimensional Wiener processes $W=\left(W_{t}\right)_{t \geq 0}$ and $B=\left(B_{t}\right)_{t \geq 0}$ are well defined. The filtration generated by $W$, together with all $\mathbb{P}$ null sets, is denoted by $\left\{\mathscr{F}_{t}\right\}_{t \geq 0}$. The $\sigma$-algebra of the predictable sets on $\Omega \times[0,+\infty)$ associated with $\left\{\mathscr{F}_{t}\right\}_{t \geq 0}$ is denoted by $\mathscr{P}$, and $\mathscr{F}:=\bigcup_{t \geq 0} \mathscr{F}_{t}$.

An Itô process (see [19]) starting from time $s$ and position $x$ is of the form

$$
\begin{aligned}
X_{t}^{s, x}= & x+\int_{s}^{t} b\left(r, X_{r}^{s, x}\right) d r+\int_{s}^{t} \sigma^{k}\left(r, X_{r}^{s, x}\right) d B_{r}^{k} \\
& +\int_{s}^{t} \theta^{k}\left(r, X_{r}^{s, x}\right) d W_{r}^{k}, \quad 0 \leq s \leq t .
\end{aligned}
$$

Here and throughout this paper, the summation over repeated indices is enforced by convention unless stated otherwise. Fix $T \in(0, \infty)$ and define

$$
u(t, x)=E_{\overline{\mathscr{F}}_{t}}\left[\int_{t}^{T} f\left(r, X_{r}^{t, x}\right) d r+G\left(X_{T}^{t, x}\right)\right], \quad(t, x) \in[0, T] \times \mathbb{R}^{d} .
$$

For the sake of convenience, we assume that:

$(\mathcal{A} 0) \quad b, \sigma, \theta$ and $f$ are $\mathscr{P} \times \mathcal{B}\left(\mathbb{R}^{d}\right)$-measurable and $G$ is $\mathscr{F}_{T} \times \mathcal{B}\left(\mathbb{R}^{d}\right)$-measurable.

Under certain conditions (see Proposition 4.4 and Remark 4.1), the random field $u$ turns out to be $\mathscr{P} \times \mathcal{B}\left(\mathbb{R}^{d}\right)$-measurable and together with some endogenous random field $v$, it satisfies the following BSPDE

$$
\left\{\begin{array}{c}
-d u(t, x)=\left[\frac{1}{2}\left(L_{k}^{2}+M_{k}^{2}\right) u(t, x)+M_{k} v^{k}(t, x)+\tilde{b}^{j}(t, x) D_{j} u(t, x)+f(t, x)\right] d t \\
-v^{r}(t, x) d W_{t}^{r}, \quad(t, x) \in[0, T] \times \mathbb{R}^{d} ; \\
u(T, x)=G(x), \\
x \in \mathbb{R}^{d},
\end{array}\right.
$$

1350-7265 C 2018 ISI/BS 
where it is written in the Hörmander form, $D=\left(D_{1}, \ldots, D_{d}\right)$ is the gradient operator, $L_{k}=$ $\sigma^{j k} D_{j}, M_{k}=\theta^{j k} D_{j}$, for $k=1, \ldots, d_{1}$, and $\tilde{b}^{j}=b^{j}-\frac{1}{2}\left(\sigma^{i k} D_{i} \sigma^{j k}+\theta^{i k} D_{i} \theta^{j k}\right)$, for $j=$ $1, \ldots, d$. BSPDE like (3) is said to be degenerate when it fails to satisfy the following superparabolicity: There exists $\lambda \in(0, \infty)$ such that

$$
\sigma^{i k} \sigma^{j k}(t, x) \xi^{i} \xi^{j} \geq \lambda|\xi|^{2} \quad \text { a.s., } \forall(t, x, \xi) \in[0, T] \times \mathbb{R}^{d} \times \mathbb{R}^{d} .
$$

Borrowing notions from the optimal stochastic control theory, we say the framework is Markovian if and only if all the coefficients $b, \sigma, \theta, f$ and $G$ are deterministic functions. In the Markovian case, $X$ is a diffusion (Markovian) process, $u$ is deterministic, $v \equiv 0$, and BSPDE (3) turns out to be a classical parabolic PDE. In Hörmander's seminal work [7], it is proved with the analytical method that given smooth coefficients $b, \sigma, \theta$ and $f$, under the hypo-ellipticity condition allowing for degenerateness (like condition $(\mathcal{H})$ below), $u$ is smooth on $[0, T) \times \mathbb{R}^{d}$, even when the terminal value $G$ is just a generalized (irregular) function. Hörmander's theorem shows in fact the smoothness of transition probabilities of hypo-elliptic diffusions, for which the probabilistic approach was formulated on the basis of Malliavin calculus (see [14]), and along this line, see $[3,15]$ and references therein for the generalizations.

In this paper, we are concerned with a Hörmander-type theorem for Itô processes, which allows random, possibly degenerate coefficients and goes beyond the scope of Markovian framework and thus of diffusion processes. In fact, for Itô processes, the smoothing property depends not only on the (hypo-)ellipticity of the diffusion coefficients but also on the extent to which the framework is Markovian. In other words, not just the degenerateness but also the randomness of coefficients may damage the smoothing property. Let us consider the following example.

Example 1.1. Let $d=d_{1}=1, \sigma \equiv 0, f \equiv 0, \theta \equiv 1, b(t, \omega)=\bar{b}\left(t, H_{t}(\omega)\right)$ and $G(x)=U(x-$ $\left.H_{T}\right) M_{T}$ with $\bar{b}$ and $U$ being deterministic functions, $H_{t}=X_{t}^{0, \eta}$ and $M_{t}=\exp \left\{\alpha W_{t}-\frac{\alpha^{2} t}{2}\right\}$ for $t \in[0, T], \eta, \alpha \in \mathbb{R}$. (In the field of mathematical finance, $X$ can be seen as a wealth process, the terminal value $G(x)=U\left(x-H_{T}\right) M_{T}$ is the utility from terminal wealth which is subject to the delivery of liability $H_{T}$, and $M_{T}$ denotes the transformation of probability measures.)

It is easy to check that $u(t, x)=U\left(x-H_{t}\right) M_{t}$ along with $v(t, x):=\alpha U\left(x-H_{t}\right) M_{t}-U^{\prime}(x-$ $\left.H_{t}\right) M_{t}$ solves BSPDE (3). Moreover, we see that $u$ does not have more spacial regularity than its terminal value $G$. Taking a close look at the non-Markovian framework, we consider the two particular cases:

(i) when $\eta=0$ and $b \equiv 0, X_{t}^{s, x}=x+W_{t}-W_{s}$ is Markovian and the framework is not Markovian due to the randomness of $G(x)=U\left(x-W_{T}\right) M_{T}$;

(ii) when $\alpha=0$ and $H$ is chosen to be the Brownian bridge with $H_{T}=0$, then $X$ is equipped with a random drift and thus is not a Markov process while the terminal value $G(x)=U(x)$ is deterministic.

In view of assumption $(\mathcal{A} 0)$, we see that the randomness of all the coefficients $b, \sigma, \theta, f$ and $G$ is only subject to the sub-filtration $\left\{\mathscr{F}_{t}\right\}_{t \geq 0}$ that is generated by Wiener process $W$ and one may conjecture that the term associated with Wiener process $B$, seen as the Markovian part, may serve to the smoothing property. The answer is affirmative. Under a hypo-ellipticity assumption on the coefficients $\left\{\sigma^{k}\right\}, k=1, \ldots, d_{1}$ (see $(\mathcal{H})$ below), we prove that the random 
field $u(t, x)$ is almost surely infinitely differentiable with respect to $x$ and each of its derivatives is continuous in $(t, x)$ on $[0, T) \times \mathbb{R}^{d}$. Compared with the time-smoothness assumption in the classical Hörmander theorem, the coefficients herein is only required to be measurable with respect to the time variable, and the time-differentiability of $u(t, x)$ is not investigated due to the appearance of the stochastic integral in BSPDE (3). For the related linear, possibly degenerate BSPDEs, a short self-contained proof is presented for the $L^{2}$-theory, and in particular, we obtain some new gradient estimates from which we start the proof of the Hörmander-type theorem.

Inspired by the filtering theory of partially observable diffusion processes, Krylov [11] has just obtained a Hörmander-type theorem for forward SPDEs. However, there is an essential difference between forward SPDEs and BSPDEs, that is, the noise term in the former is exogenous, while in the latter it comes from the martingale representation and is governed by the coefficients, and thus it is endogenous. On the other hand, we would also emphasise that the method of Krylov [11] relies on the generalized Itô-Wentzell formula and associated results on deterministic PDEs, while we use directly elaborate estimates on solutions of degenerate BSPDEs.

The study of linear BSPDEs can date back to about thirty years ago (see [2]). They arise in many applications of probability theory and stochastic processes, for instance, in the nonlinear filtering and stochastic control theory for processes with incomplete information, as an adjoint equation of the Duncan-Mortensen-Zakai filtration equation (for instance, see [2,9]). Naturally in the dynamic programming theory, a class of nonlinear BSPDEs as the so-called stochastic Hamilton-Jacobi-Bellman equations, are introduced in the study of non-Markovian control problems (see [16]). In addition, the representation relationship between forward-backward stochastic differential equations and BSPDEs yields the stochastic Feynman-Kac formula (see [9]). The BSPDEs have already received extensive attention; see $[1,6,10,17,18,20]$ and references therein for the recent developments.

The rest of this paper is organized as follows. In Section 2, we introduce some notations and state the main result (Theorem 2.2). Section 3 is devoted to an $L^{2}$-theory for linear degenerate BSPDEs. In Section 4, we prove the Hörmander-type theorem.

\section{Preliminaries and main results}

For each $l \in \mathbb{N}^{+}$and domain $\Pi \subset \mathbb{R}^{l}$, denote by $C_{c}^{\infty}(\Pi)$ the space of infinitely differentiable functions with compact supports in $\Pi$. $L^{2}\left(\mathbb{R}^{d}\right)$ ( $L^{2}$ for short) is the usual Lebesgue integrable space with usual scalar product $\langle\cdot, \cdot\rangle$ and norm $\|\cdot\|$. For $n \in(-\infty, \infty)$, we denote by $H^{n}$ the space of Bessel potentials, that is $H^{n}:=(1-\Delta)^{-n / 2} L^{2}$ with the Sobolev norm

$$
\|\phi\|_{n}:=\left\|(1-\Delta)^{n / 2} \phi\right\|_{L^{2}}, \quad \phi \in H^{n} .
$$

For the sake of convenience, we shall also use $\langle\cdot, \cdot\rangle$ to denote the duality between $\left(H^{n}\right)^{k}$ and $\left(H^{-n}\right)^{k}\left(k \in \mathbb{N}^{+}, n \in \mathbb{R}\right)$ as well as that between the Schwarz function space $\mathscr{D}$ and $C_{c}^{\infty}\left(\mathbb{R}^{d}\right)$. Moreover, We always omit the index associated to the dimension when there is no confusion.

Given Banach space $\left(\mathbb{B},\|\cdot\|_{\mathbb{B}}\right), \mathcal{S}^{2}(\mathbb{B})$ is the set of all the $\mathbb{B}$-valued, $\left(\mathscr{F}_{t}\right)$-adapted and continuous processes $\left(X_{t}\right)_{t \in[0, T]}$ such that

$$
\|X\|_{\mathcal{S}^{2}(\mathbb{B})}:=\left\|\sup _{t \in[0, T]}\right\| X_{t}\left\|_{\mathbb{B}}\right\|_{L^{2}(\Omega)}<\infty .
$$


For $p \in[1, \infty]$, denote by $\mathcal{L}^{p}(\mathbb{B})$ the totality of all the $\mathbb{B}$-valued, $\left(\mathscr{F}_{t}\right)$-adapted processes $\left(X_{t}\right)_{t \in[0, T]}$ such that

$$
\|X\|_{\mathcal{L}^{p}(V)}:=\|\| X_{t}\left\|_{\mathbb{B}}\right\|_{L^{p}(\Omega \times[0, T])}<\infty .
$$

Obviously, both $\left(\mathcal{S}^{2}(\mathbb{B}),\|\cdot\|_{\mathcal{S}^{2}(\mathbb{B})}\right)$ and $\left(\mathcal{L}^{p}(\mathbb{B}),\|\cdot\|_{\mathcal{L}^{p}(\mathbb{B})}\right)$ are Banach spaces.

Denote by $C_{b}$ the space of bounded continuous functions on $\mathbb{R}^{d}$ equipped with the usual uniform norm $\|\cdot\|_{\infty}$. Let $C_{b}^{\infty}$ be the set of infinitely differentiable functions with bounded derivatives of any order. Denote by $\mathcal{L}^{\infty}\left(C_{b}^{\infty}\right)$ the set of functions $h$ on $\Omega \times[0, T] \times \mathbb{R}^{d}$ such that $h(t, x)$ is infinitely differentiable with respect to $x$ and all the derivatives of any order belong to $\mathcal{L}^{\infty}\left(C_{b}\right)$.

Throughout this work, we denote $I^{n}=(1-\Delta)^{n / 2}$ for $n \in \mathbb{R}$. Then $I^{n}$ belongs to $\Psi_{n}$ that is the class of pseudo-differential operators of order $n$. By the pseudo-differential operator theory (see [8] for instance), the $m$-th order differential operator belongs to $\Psi_{m}$ for $m \in \mathbb{N}^{+}$, the multiplication by elements of $C_{b}^{\infty}$ lies in $\Psi_{0}$, and for the reader's convenience, some useful basic results are collected below.

Lemma 2.1. (i) If $J_{1} \in \Psi_{n_{1}}$ and $J_{2} \in \Psi_{n_{2}}$ with $n_{1}, n_{2} \in \mathbb{R}$, then $J_{1} J_{2} \in \Psi_{n_{1}+n_{2}}$ and the Lie bracket $\left[J_{1}, J_{2}\right]:=J_{1} J_{2}-J_{2} J_{1} \in \Psi_{n_{1}+n_{2}-1}$.

(ii) For $m \in(0, \infty)$, let $\zeta$ belong to the continuous function space $C_{b}^{m}$ which is defined as usual. Then for any $n \in(-m, m)$ there exists constant $C$ such that

$$
\|\zeta \phi\|_{n} \leq C\|\zeta\|_{C^{m}}\|\phi\|_{n} \quad \forall \phi \in H^{n}
$$

Set

$$
\mathbb{V}_{0}=\left\{L_{1}, \ldots, L_{d_{1}}\right\} \quad \text { and } \quad \mathbb{V}_{n+1}=\mathbb{V}_{n} \cup\left\{\left[L_{k}, V\right]: V \in \mathbb{V}_{n}, k=1, \ldots, d_{1}\right\}
$$

Denote by $\mathbb{L}_{n}$ the set of linear combinations of elements of $\mathbb{V}_{n}$ with coefficients of $\mathcal{L}^{\infty}\left(C_{b}^{\infty}\right)$. We then introduce the following Hörmander-type condition.

(H) There exists $n_{0} \in \mathbb{N}_{0}$ such that $\left\{D_{i}: i=1, \ldots, d\right\} \subset \mathbb{L}_{n_{0}}$.

Throughout this paper, we denote $\eta=2^{-n_{0}}$.

Instead of BSPDE (3), we consider the following one of the general form

$$
\left\{\begin{array}{cc}
-d u(t, x)=\left[\left(\frac{1}{2} L_{k}^{2}+\frac{1}{2} M_{k}^{2}\right) u+M_{k} v^{k}+b^{j} D_{j} u+c u+\gamma^{l} v^{l}+f+L_{k} g^{k}\right](t, x) d t & (t, x) \in Q ; \\
-v^{r}(t, x) d W_{t}^{r}, & x \in \mathbb{R}^{d} .
\end{array}\right.
$$

We define the following assumption.

$(\mathcal{A} 1)$ For $i=1, \ldots, d, k=1, \ldots, d_{1}, \sigma^{i k}, \theta^{i k}, b^{i}, \gamma^{k}, c \in \mathcal{L}^{\infty}\left(C_{b}^{\infty}\right)$.

Definition 2.1. A pair of processes $(u, v)$ is called a solution to BSPDE (4) if $(u, v) \in \mathcal{S}^{2}\left(H^{m}\right) \times$ $\mathcal{L}^{2}\left(H^{m-1}\right)$ for some $m \in \mathbb{R}$ and BSPDE (3) holds in the distributional sense, i.e., for any $\zeta \in$ 
$C_{c}^{\infty}(\mathbb{R}) \otimes C_{c}^{\infty}\left(\mathbb{R}^{d}\right)$ there holds almost surely

$$
\begin{aligned}
& \langle\zeta(t), u(t)\rangle-\langle\zeta(T), G\rangle+\int_{t}^{T}\left\langle\partial_{s} \zeta(s), u(s)\right\rangle d s+\int_{t}^{T}\left\langle\zeta(s), v^{r}(s)\right\rangle d W_{s}^{r} \\
& \quad=\int_{t}^{T}\left\langle\zeta, \frac{1}{2}\left(L_{k}^{2}+M_{k}^{2}\right) u+M_{k} v^{k}+b^{j} D_{j} u+c u+\gamma^{l} v^{l}+f+L_{k} g^{k}\right\rangle(s) d s \\
& \forall t \in[0, T] .
\end{aligned}
$$

We now state our main result. The following theorem is a summary of Theorem 3.3, Corollary 4.3 and Theorem 4.1 .

Theorem 2.2. Let assumption (A1) hold. Assume $(f, g, G) \in \mathcal{L}^{2}\left(H^{m}\right) \times \mathcal{L}^{2}\left(\left(H^{m}\right)^{d_{1}}\right) \times$ $L^{2}\left(\Omega, \mathscr{F}_{T} ; H^{m}\right)$, for some $m \in \mathbb{R}$. There hold the following three assertions:

(i) BSPDE (4) admits a unique solution $(u, v) \in \mathcal{S}^{2}\left(H^{m}\right) \times \mathcal{L}^{2}\left(H^{m-1}\right)$ with $\left(L_{k} u, v^{k}+\right.$ $\left.M_{k} u\right) \in \mathcal{L}^{2}\left(H^{m}\right) \times \mathcal{L}^{2}\left(H^{m}\right), k=1, \ldots, d_{1}$, and

$$
\begin{aligned}
& E \sup _{t \in[0, T]}\|u(t)\|_{m}^{2}+E \int_{0}^{T}\left(\sum_{k=1}^{d_{1}}\left\|L_{k} u(t)\right\|_{m}^{2}+\|v(t)+D u(t) \theta(t)\|_{m}^{2}\right) d t \\
& \quad \leq C\left\{E\|G\|_{m}^{2}+E \int_{0}^{T}\left(\|f(s)\|_{m}^{2}+\|g(s)\|_{m}^{2}\right) d s\right\},
\end{aligned}
$$

with $C$ depending on $T, m$ and quantities related to coefficients $\sigma, \theta, b, c$ and $\gamma$.

(ii) If the Hörmander-type condition $(\mathcal{H})$ holds, for the above solution $(u, v)$, we have further

$$
E \int_{0}^{T}\|u(t)\|_{m+\eta}^{2} d t \leq C\left\{E\|G\|_{m}^{2}+E \int_{0}^{T}\left(\|f(s)\|_{m}^{2}+\|g(s)\|_{m}^{2}\right) d s\right\},
$$

with $C$ depending on $T, m, n_{0}, \sigma, \theta, b, c$ and $\gamma$.

(iii) If both $(f, g) \in \bigcap_{n \in \mathbb{R}}\left(\mathcal{L}^{2}\left(H^{n}\right) \times \mathcal{L}^{2}\left(\left(H^{n}\right)^{d_{1}}\right)\right)$ and assumption $(\mathcal{H})$ hold, we have for each $\varepsilon \in(0, T)$,

$$
(u, v) \in \bigcap_{n \in \mathbb{R}} L^{2}\left(\Omega ; C\left([0, T-\varepsilon] ; H^{n}\right)\right) \times L^{2}\left(\Omega ; L^{2}\left(0, T-\varepsilon ; H^{n-1}\right)\right),
$$

and for any $n \in \mathbb{R}$

$$
\begin{aligned}
& E \sup _{t \in[0, T-\varepsilon]}\|u(t)\|_{n}^{2}+E \int_{0}^{T-\varepsilon}\left(\|u(t)\|_{n+\eta}^{2}+\|v(t)+D u(t) \theta(t)\|_{n}^{2}\right) d t \\
& \quad \leq C\left\{E\|G\|_{m}^{2}+E \int_{0}^{T}\left(\|f(s)\|_{n}^{2}+\|g(s)\|_{n}^{2}\right) d s\right\},
\end{aligned}
$$


with the constant $C$ depending on $\varepsilon, T, n, m, n_{0}, \sigma, \theta, \gamma, b$ and $c$. In particular, the random field $u(t, x)$ is infinitely differentiable with respect to $x$ on $[0, T) \times \mathbb{R}^{d}$ and each derivative is a continuous function on $[0, T) \times \mathbb{R}^{d}$.

Remark 2.1. An $L^{2}$-theory on degenerate BSPDEs was initiated by Zhou [21], and it was developed recently by $[5,9,13]$. Along this line, to get a solution of BSPDE (4) in space $\mathcal{S}^{2}\left(H^{m}\right) \times \mathcal{L}^{2}\left(H^{m-1}\right)$ requires that $L_{k} g^{k}$ lies in $\mathcal{L}^{2}\left(H^{m}\right)$ for some $m \in \mathbb{N}^{+}$, but in (i) of Theorem 2.2, $g^{k}$ is allowed to be in $\mathcal{L}^{2}\left(H^{m}\right)$ and thus $L_{k} g^{k} \in \mathcal{L}^{2}\left(H^{m-1}\right)$, and there holds the additional gradient estimate $L_{k} u \in \mathcal{L}^{2}\left(H^{m}\right)$, for $k=1, \ldots, d_{1}$. Moreover, compared with the existing $L^{2}$-theory on degenerate BSPDEs, $m$ herein can be any real number instead of being restricted to positive integers, and under the Hörmander-type condition $(\mathcal{H})$, one further has $u \in \mathcal{L}^{2}\left(H^{m+\eta}\right)$ in (ii). Hence, the $L^{2}$-theory presented in (i) and (ii) of Theorem 2.2 seems to be of independent interest.

Starting from the estimate of $L_{k} u$, we prove the Hörmander-type theorem ((iii) of Theorem 2.2) by increasing the regularity of $u$ step by step. In this paper, it is indeed necessary to allow $m$ to be real number in the $L^{2}$-theory, as for each step the regularity is increased from $m$ to $m+\varepsilon$ for a possibly real number $\varepsilon \in(0,1]$ (see Section 4 below for more details).

\section{An $L^{2}$ theory of linear BSPDEs}

We consider the following BSPDE

$$
\left\{\begin{array}{cc}
-d u(t, x)=\left[\frac{1}{2}\left(L_{k}^{2}+M_{k}^{2}\right) u+M_{k} v^{k}+b^{j} D_{j} u+c u+\gamma^{l} v^{l}+f+L_{k} g^{k}\right](t, x) d t \\
+\delta \Delta u(t, x) d t-v^{r}(t, x) d W_{t}^{r}, & (t, x) \in Q ; \\
u(T, x)=G(x), & x \in \mathbb{R}^{d},
\end{array}\right.
$$

with $\delta \geq 0$.

Note that we do not need the Hörmander-type condition $(\mathcal{H})$ in this section. We would first give an a priori estimate on the solution for BSPDE (7).

Proposition 3.1. Let assumption ( $\mathcal{A} 1)$ hold. For $(f, g, G) \in \mathcal{L}^{2}\left(H^{m}\right) \times \mathcal{L}^{2}\left(\left(H^{m}\right)^{d_{1}}\right) \times L^{2}(\Omega$, $\left.\mathscr{F}_{T} ; H^{m}\right)$ with $m \in \mathbb{R}$, if $(u, v) \in\left(\mathcal{S}^{2}\left(H^{m+1}\right) \cap \mathcal{L}^{2}\left(H^{m+2}\right)\right) \times \mathcal{L}^{2}\left(\left(H^{m+1}\right)^{d_{1}}\right)$ is a solution of BSPDE (7), then one has

$$
\begin{aligned}
& E \sup _{t \in[0, T]}\|u(t)\|_{m}^{2}+E \int_{0}^{T}\left(\delta\|D u(t)\|_{m}^{2}+\sum_{k=1}^{d_{1}}\left\|L_{k} u(t)\right\|_{m}^{2}+\|v(t)+D u(t) \theta(t)\|_{m}^{2}\right) d t \\
& \quad \leq C\left\{E\|G\|_{m}^{2}+E \int_{0}^{T}\left(\|f(s)\|_{m}^{2}+\|g(s)\|_{m}^{2}\right) d s\right\},
\end{aligned}
$$

with $C$ depends only on $T, m, \sigma, \theta, \gamma, b$ and $c$.

Proof. Set $\xi=v+D u \theta$. Putting $L_{k}^{\prime}:=D_{i}\left(\sigma^{i k} \cdot\right)$ and $M_{k}^{\prime}=D_{i}\left(\theta^{i k} \cdot\right)$, we have $L_{k}=L_{k}^{\prime}+c_{k}$ and $M_{k}=M_{k}^{\prime}+\alpha_{k}$ with $c_{k}=-\left(D_{i} \sigma^{i k}\right) \cdot$ and $\alpha_{k}=-\left(D_{i} \theta^{i k}\right)$, for $k=1, \ldots, d_{1}$. Applying Itô 
formula for the square norm (see, e.g., [12], Theorem 3.1), one has almost surely for $t \in[0, T]$,

$$
\begin{aligned}
& \left\|I^{m} u(t)\right\|^{2}+\int_{t}^{T}\left(2 \delta\left\|I^{m} D u(s)\right\|^{2}+\left\|I^{m}(\xi-D u \theta)(s)\right\|^{2}\right) d s \\
& =\left\|I^{m} G\right\|^{2}+\int_{t}^{T}\left\langle I^{m} u(s), I^{m}\left(\left(L_{k}^{2}+M_{k}^{2}\right) u+2 M_{k}\left(\xi^{k}-D_{i} u \theta^{i k}\right)\right)(s)\right\rangle d s \\
& \quad+\int_{t}^{T} 2\left\langle I^{m} u(s), I^{m}\left(b^{j} D_{j} u+\gamma^{l}\left(\xi^{l}-D_{i} u \theta^{i l}\right)+c u+f+L_{k} g\right)(s)\right\rangle d s \\
& \quad-\int_{t}^{T} 2\left\langle I^{m} u(s), I^{m}(\xi-D u \theta)(s) d W_{s}\right\rangle .
\end{aligned}
$$

First, basic calculations yield

$$
\begin{aligned}
&\left\langle I^{m} u, I^{m}\left(L_{k}^{2} u\right)\right\rangle \\
&=\left\langle I^{m} u, I^{m}\left(L_{k}^{\prime}+c_{k}\right) L_{k} u\right\rangle \\
&=-\left\langle L_{k} I^{m} u, I^{m} L_{k} u\right\rangle+\left\langle I^{m} u,\left[I^{m}, L_{k}^{\prime}\right] L_{k} u+I^{m} c_{k} L_{k} u\right\rangle \\
&=-\left\|I^{m} L_{k} u\right\|^{2}+\left\langle\left[I^{m}, L_{k}\right] u, I^{m} L_{k} u\right\rangle+\left\langle I^{m} u,\left[I^{m}, L_{k}^{\prime}\right] L_{k} u+I^{m} c_{k} L_{k} u\right\rangle \\
& \leq-(1-\varepsilon)\left\|I^{m} L_{k} u\right\|^{2}+C_{\varepsilon}\left\|I^{m} u\right\|^{2}, \quad \varepsilon \in(0,1), \\
&\left\langle I^{m} u, I^{m}\left(\gamma^{l} \xi^{l}+c u+f+L_{k} g^{k}\right)\right\rangle \\
&=\left\langle I^{m} u, I^{m}\left(\gamma^{l} \xi^{l}+c u+f\right)\right\rangle+\left\langle I^{m} u,\left(L_{k} I^{m}+\left[I^{m}, L_{k}\right]\right) g^{k}\right\rangle \\
&=\left\langle I^{m} u, I^{m}\left(\gamma^{l} \xi^{l}+c u+f\right)\right\rangle-\left\langle I^{m} L_{k} u+\left[L_{k}, I^{m}\right] u, I^{m} g^{k}\right\rangle \\
& \quad+\left\langle I^{m} u,\left(\alpha_{k} I^{m}+\left[I^{m}, L_{k}\right]\right) g^{k}\right\rangle \\
& \leq \varepsilon\left(\left\|I^{m} L_{k} u\right\|^{2}+\left\|I^{m} \xi^{l}\right\|^{2}\right)+C_{\varepsilon}\left(\left\|I^{m} u\right\|^{2}+\left\|I^{m} f\right\|^{2}+\left\|I^{m} g^{k}\right\|^{2}\right), \quad \varepsilon \in(0,1)
\end{aligned}
$$

and

$$
\begin{aligned}
&\left\langle I^{m} u, I^{m}\left(M_{k}^{2} u+2 M_{k}\left(\xi^{k}-D_{i} u \theta^{i k}\right)\right)\right\rangle \\
&=\left\langle I^{m} u, I^{m}\left(-M_{k}^{2} u+2 M_{k} \xi^{k}\right)\right\rangle \\
&=\left\langle I^{m} u,-M_{k}^{\prime} I^{m} M_{k} u+\left[M_{k}^{\prime}, I^{m}\right] M_{k} u\right\rangle+\left\langle I^{m} u, I^{m}\left(2 M_{k}^{\prime} \xi^{k}+2 \alpha_{k} \xi^{k}-\alpha_{k} M_{k} u\right)\right\rangle \\
&=\left\langle M_{k} I^{m} u, I^{m} M_{k} u\right\rangle+\left\langle I^{m} u,\left[M_{k}^{\prime}, I^{m}\right] M_{k} u\right\rangle \\
& \quad-2\left\langle M_{k} I^{m} u, I^{m} \xi^{k}\right\rangle+2\left\langle I^{m} u,\left[I^{m}, M_{k}^{\prime}\right] \xi^{k}\right\rangle \\
& \quad+\left\langle I^{m} u, I^{m}\left(2 \alpha_{k} \xi^{k}-\alpha_{k} M_{k} u\right)\right\rangle \\
&=\left\|I^{m} M_{k} u\right\|^{2}-2\left\langle I^{m} M_{k} u, I^{m} \xi^{k}\right\rangle+\left\langle\left[M_{k}, I^{m}\right] u, I^{m} M_{k} u\right\rangle+\left\langle I^{m} u,\left[M_{k}^{\prime}, I^{m}\right] M_{k} u\right\rangle
\end{aligned}
$$




$$
\begin{aligned}
& -2\left\langle\left[M_{k}, I^{m}\right] u, I^{m} \xi^{k}\right\rangle+2\left\langle I^{m} u,\left[I^{m}, M_{k}^{\prime}\right] \xi^{k}\right\rangle+\left\langle I^{m} u, I^{m}\left(2 \alpha_{k} \xi^{k}-\alpha_{k} M_{k} u\right)\right\rangle \\
= & \left\|I^{m} M_{k} u\right\|^{2}-2\left\langle I^{m} M_{k} u, I^{m} \xi^{k}\right\rangle-\left\|\left[M_{k}, I^{m}\right] u\right\|^{2} \\
& +\left\langle\left[M_{k}, I^{m}\right] u, M_{k} I^{m} u\right\rangle+\left\langle I^{m} u,\left[M_{k}^{\prime}, I^{m}\right] M_{k} u\right\rangle-\left\langle I^{m} u, \alpha_{k} M_{k} I^{m} u\right\rangle \\
& -2\left\langle\left[M_{k}, I^{m}\right] u, I^{m} \xi^{k}\right\rangle+2\left\langle I^{m} u,\left[I^{m}, M_{k}^{\prime}\right] \xi^{k}\right\rangle+\left\langle I^{m} u, I^{m}\left(2 \alpha_{k} \xi^{k}\right)+\left[\alpha_{k} M_{k}, I^{m}\right] u\right\rangle \\
= & \left\|I^{m} M_{k} u\right\|^{2}-2\left\langle I^{m} M_{k} u, I^{m} \xi^{k}\right\rangle-\left\|\left[M_{k}, I^{m}\right] u\right\|^{2} \\
& +\left\langle I^{m} u, \alpha_{k}\left[M_{k}, I^{m}\right] u-\left[\alpha_{k}, I^{m}\right] M_{k} u\right\rangle \\
& -\left\langle I^{m} u, \alpha_{k} M_{k} I^{m} u\right\rangle+\left\langle I^{m} u,\left[\left[M_{k}, I^{m}\right], M_{k}\right] u\right\rangle \\
& -2\left\langle\left[M_{k}, I^{m}\right] u, I^{m} \xi^{k}\right\rangle+2\left\langle I^{m} u,\left[I^{m}, M_{k}\right] \xi^{k}\right\rangle+\left\langle I^{m} u, 2 \alpha_{k} I^{m} \xi^{k}+\left[\alpha_{k} M_{k}, I^{m}\right] u\right\rangle \\
\leq & \left\|I^{m} M_{k} u\right\|^{2}-2\left\langle I^{m} M_{k} u, I^{m} \xi^{k}\right\rangle-\left\|\left[M_{k}, I^{m}\right] u\right\|^{2} \\
& +\varepsilon\left\|I^{m} \xi\right\|^{2}+C_{\varepsilon}\left\|I^{m} u\right\|^{2}, \quad \varepsilon \in(0,1),
\end{aligned}
$$

where we have used the relation

$$
\left\langle I^{m} u, \alpha_{k} M_{k} I^{m} u\right\rangle=-\frac{1}{2}\left\langle I^{m} u, D_{i}\left(\alpha_{k} \theta^{i k}\right) I^{m} u\right\rangle .
$$

Noticing relations like (13) and that for $i=1, \ldots, d, k=1, \ldots, d_{1}$,

$$
\begin{aligned}
\left\|I^{m}\left(\xi^{k}-M_{k} u\right)\right\|^{2} & =\left\|I^{m} \xi^{k}\right\|^{2}-2\left\langle I^{m} \xi^{k}, I^{m} M_{k} u\right\rangle+\left\|I^{m} M_{k} u\right\|^{2}, \\
\left\langle I^{m} u, I^{m}\left(\gamma^{k} D_{i} u \theta^{i k}\right)\right\rangle & =\frac{1}{2}\left\langle I^{m} u, D_{i}\left(\gamma^{k} \theta^{i k}\right) I^{m} u+2\left[\gamma^{k} \theta^{i k} D_{i}, I^{m}\right] u\right\rangle, \\
\left\langle I^{m} u, I^{m}\left(b^{i} D_{i} u\right)\right\rangle & =-\frac{1}{2}\left\langle I^{m} u, D_{i}\left(b^{i}\right) I^{m} u+2\left[b^{i} D_{i}, I^{m}\right] u\right\rangle,
\end{aligned}
$$

putting (9), (10), (11) and (12) together, and taking expectations on both sides of (9), one gets by Gronwall inequality

$$
\begin{aligned}
& \sup _{t \in[0, T]} E\|u(t)\|_{m}^{2}+E \int_{0}^{T}\left(\delta\|D u(t)\|_{m}^{2}+\sum_{k=1}^{d_{1}}\left\|L_{k} u(t)\right\|_{m}^{2}+\|v(t)+D u(t) \theta(t)\|_{m}^{2}\right) d t \\
& \quad \leq C\left\{E\|G\|_{m}^{2}+E \int_{0}^{T}\left(\|f(s)\|_{m}^{2}+\|g(s)\|_{m}^{2}\right) d s\right\} .
\end{aligned}
$$

On the other hand, one has for each $t \in[0, T)$

$$
\begin{aligned}
& E \sup _{\tau \in[t, T]}\left|\int_{\tau}^{T} 2\left\langle I^{m} u(s), I^{m}(\xi-D u \theta)(s) d W_{s}\right\rangle\right| \\
& \leq 2 E \sup _{\tau \in[t, T]}\left|\int_{t}^{\tau} 2\left\langle I^{m} u(s), I^{m}(\xi-D u \theta)(s) d W_{s}\right\rangle\right|
\end{aligned}
$$




$$
\begin{aligned}
& \leq C\left(E \sum_{k=1}^{d_{1}} \int_{t}^{T}\left(\left|\left\langle I^{m} u(s), I^{m} \xi^{k}(s)\right\rangle\right|^{2}+\left|\left\langle I^{m} u(s),\left(M_{k} I^{m}+\left[I^{m}, M_{k}\right]\right) u(s)\right\rangle\right|^{2}\right) d s\right)^{1 / 2} \\
& \leq C\left(E \int_{t}^{T}\left(\left\|I^{m} u(s)\right\|^{2}\left\|I^{m} \xi(s)\right\|^{2}+\left\|I^{m} u(s)\right\|^{4}\right) d s\right)^{1 / 2} \\
& \leq \varepsilon E \sup _{s \in[t, T]}\left\|I^{m} u(s)\right\|^{2}+C_{\varepsilon} E \int_{t}^{T}\left(\left\|I^{m} \xi(s)\right\|^{2}+\left\|I^{m} u(s)\right\|^{2}\right) d s \quad \forall \varepsilon \in(0,1),
\end{aligned}
$$

which together with (9), (10), (12) and (14) implies (8).

An immediate consequence of Proposition 3.1 is the following.

Corollary 3.2. Let assumption $(\mathcal{A} 1)$ hold. For $(f, g, G) \in \mathcal{L}^{2}\left(H^{m}\right) \times \mathcal{L}^{2}\left(\left(H^{m}\right)^{d_{1}}\right) \times L^{2}(\Omega$, $\left.\mathscr{F}_{T} ; H^{m}\right)$ with $m \in \mathbb{R}$, the solution of BSPDE (7) is unique.

Theorem 3.3. Let assumption ( $\mathcal{A} 1)$ hold. Given $(f, g, G) \in \mathcal{L}^{2}\left(H^{m}\right) \times \mathcal{L}^{2}\left(\left(H^{m}\right)^{d_{1}}\right) \times L^{2}(\Omega$, $\left.\mathscr{F}_{T} ; H^{m}\right)$ with $m \in \mathbb{R}, B S P D E(4)$ (equivalently, BSPDE (7) with $\delta=0$ ) admits a unique solution $(u, v) \in \mathcal{S}^{2}\left(H^{m}\right) \times \mathcal{L}^{2}\left(H^{m-1}\right)$ with $\left(L_{k} u, v^{k}+M_{k} u\right) \in \mathcal{L}^{2}\left(H^{m}\right) \times \mathcal{L}^{2}\left(H^{m}\right), k=1, \ldots, d_{1}$, and

$$
\begin{aligned}
& E \sup _{t \in[0, T]}\|u(t)\|_{m}^{2}+E \int_{0}^{T}\left(\sum_{k=1}^{d_{1}}\left\|L_{k} u(t)\right\|_{m}^{2}+\|v(t)+D u(t) \theta(t)\|_{m}^{2}\right) d t \\
& \quad \leq C\left\{E\|G\|_{m}^{2}+E \int_{0}^{T}\left(\|f(s)\|_{m}^{2}+\|g(s)\|_{m}^{2}\right) d s\right\}
\end{aligned}
$$

with $C$ depending on $T, m, \sigma, \theta, \gamma, b$ and $c$.

Proof. We use the method of approximation. Choose $\left\{\delta_{l}\right\}_{l \in \mathbb{N}^{+}} \subset(0,1)$ and

$$
\left\{\left(f_{n}, g_{n}, G_{n}\right)\right\}_{n \in \mathbb{N}^{+}} \subset \mathcal{L}^{2}\left(H^{m+5}\right) \times \mathcal{L}^{2}\left(\left(H^{m+5}\right)^{d_{1}}\right) \times L^{2}\left(\Omega, \mathscr{F}_{T} ; H^{m+5}\right)
$$

such that $\delta_{l}$ converges down to 0 and $\left(f_{n}, g_{n}, G_{n}\right)$ converges to $(f, g, G)$ in $\mathcal{L}^{2}\left(H^{m}\right) \times$ $\mathcal{L}^{2}\left(\left(H^{m}\right)^{d_{1}}\right) \times L^{2}\left(\Omega, \mathscr{F}_{T} ; H^{m}\right)$. By the $L^{p}$-theory of BSPDEs (see [4] for instance), BSPDE (7) admits a unique solution $\left(u_{l, n}, v_{l, n}\right) \in\left(\mathcal{S}^{2}\left(H^{m+5}\right) \cap \mathcal{L}^{2}\left(H^{m+6}\right)\right) \times \mathcal{L}^{2}\left(H^{m+5}\right)$ associated with $\left(\delta_{l}, f_{n}, g_{n}, G_{n}\right)$.

For each $n$, it follows from Proposition 3.1 that $\left\{\left(u_{l, n}, L_{k} u_{l, n}, v_{l, n}+D u_{l, n} \theta\right)\right\}_{l \in \mathbb{N}^{+}}$is bounded in $\mathcal{S}^{2}\left(H^{m+4}\right) \times \mathcal{L}^{2}\left(H^{m+4}\right) \times \mathcal{L}^{2}\left(\left(H^{m+4}\right)^{d_{1}}\right), k=1, \ldots, d_{1}$. Notice that $\delta_{l} \Delta u_{l, n}$ tends to zero in $\mathcal{L}^{2}\left(H^{m+2}\right)$ as $l$ goes to infinity. Therefore, letting $l$ tend to infinity, from Proposition 3.1 and Corollary 3.2 we derive the unique solution $\left(u_{n}, v_{n}\right)$ for BSPDE (7) associated with $\left(f_{n}, g_{n}, G_{n}\right)$ and $\delta=0$ such that $\left(u_{n}, L_{k} u_{n}, v_{n}+D u_{n} \theta\right) \in \mathcal{S}^{2}\left(H^{m+2}\right) \times \mathcal{L}^{2}\left(H^{m+2}\right) \times \mathcal{L}^{2}\left(\left(H^{m+2}\right)^{d_{1}}\right)$ for $k=1, \ldots, d_{1}$.

Furthermore, letting $n$ go to infinity, again by Proposition 3.1 and Corollary 3.2, one obtains the unique solution $(u, v)$ and associated estimates. This completes the proof. 
Remark 3.1. Like in [5,9], the random field $v+D u \theta \in \mathcal{L}^{2}\left(\left(H^{m}\right)^{d_{1}}\right)$ is estimated as a unity which appears in the corresponding BSDE (see (22) below for instance), and thus we only have $v \in$ $\mathcal{L}^{2}\left(H^{m-1}\right)$ (see Example 1.1). In fact, if we further have $\sigma \sigma^{\mathcal{T}} \geq \theta \theta^{\mathcal{T}}$, then $D u \theta \in \mathcal{L}^{2}\left(H^{m}\right)$ and thus $v \in \mathcal{L}^{2}\left(H^{m}\right)$, as $u, L_{k} u \in \mathcal{L}^{2}\left(H^{m}\right), k=1, \ldots, d_{1}$. In addition, in view of (ii) of Lemma 2.1 and the proofs involved in this section, the required regularity for the coefficients $b, c, \sigma, \theta$ and $\gamma$ can be relaxed like in [5,9], but we would not seek such a generality in the present paper.

\section{Hörmander-type theorem}

Recall that $\eta=2^{-n_{0}}$. Basing on the $L^{2}$-theory of SPDEs presented in the preceding section, we derive the following Hörmander-type theorem.

Theorem 4.1. Let assumptions $(\mathcal{A} 1)$ and $(\mathcal{H})$ hold. Suppose that

$$
(f, g) \in \bigcap_{n \in \mathbb{R}}\left(\mathcal{L}^{2}\left(H^{n}\right) \times \mathcal{L}^{2}\left(\left(H^{n}\right)^{d_{1}}\right)\right) \quad \text { and } \quad G \in L^{2}\left(\Omega, \mathscr{F}_{T} ; H^{m}\right) \quad \text { for some } m \in \mathbb{R} .
$$

For the unique solution $(u, v)$ of BSPDE (4) in Theorem 3.3, we have for any $\varepsilon \in(0, T)$

$$
(u, v) \in \bigcap_{n \in \mathbb{R}} L^{2}\left(\Omega ; C\left([0, T-\varepsilon] ; H^{n}\right)\right) \times L^{2}\left(\Omega ; L^{2}\left(0, T-\varepsilon ; H^{n-1}\right)\right),
$$

and for any $n \in \mathbb{R}$

$$
\begin{aligned}
& E \sup _{t \in[0, T-\varepsilon]}\|u(t)\|_{n}^{2}+E \int_{0}^{T-\varepsilon}\left(\|u(t)\|_{n+\eta}^{2}+\|v(t)+D u(t) \theta(t)\|_{n}^{2}\right) d t \\
& \quad \leq C\left\{E\|G\|_{m}^{2}+E \int_{0}^{T}\left(\|f(s)\|_{n}^{2}+\|g(s)\|_{n}^{2}\right) d s\right\},
\end{aligned}
$$

with the constant $C$ depending on $\varepsilon, T, n, m, n_{0}, \sigma, \theta, \gamma, b$ and $c$. In particular, the random field $u(t, x)$ is almost surely infinitely differentiable with respect to $x$ on $[0, T) \times \mathbb{R}^{d}$ and each derivative is a continuous function on $[0, T) \times \mathbb{R}^{d}$.

Because of the appearance of the stochastic integral in BSPDE (4), we do not investigate the time-differentiability of $u(t, x)$ and the coefficients herein is only required to be measurable with respect to the time variable, while in the classical Hörmander theorem, the associated coefficients are smooth and the function $u(t, x)$ turns out to be deterministic and smooth with respect to the time variable.

Before the proof of Theorem 4.1, we first give an estimate on the Lie bracket.

Lemma 4.2. For $\{\tilde{L}, L\} \subset \bigcup_{l \geq 0} \mathbb{V}_{l}, m \in \mathbb{R}$ and $\varepsilon \in[0,1]$, there exists a positive constant $C$ such that almost surely for any $\phi \in H^{m}$ with $\widetilde{L} \phi \in H^{m-1+\varepsilon}$ and $L \phi \in H^{m}$,

$$
\|[\widetilde{L}, L] \phi\|_{m-1+\varepsilon / 2} \leq C\left(\|\widetilde{L} \phi\|_{m-1+\varepsilon}+\|L \phi\|_{m}+\|\phi\|_{m}\right) .
$$




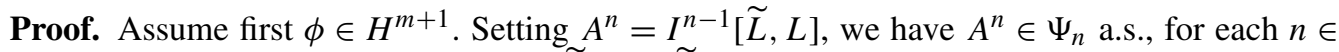
$\mathbb{R}$. As the joint operators of $H$ and $L, \widetilde{L}^{*}=-\widetilde{L}+\tilde{c}$ and $L^{*}=-L+\bar{c}$ with $\tilde{c}, \bar{c} \in \mathcal{L}^{\infty}\left(C_{b}^{\infty}\right)$, respectively. By Lemma 2.1, one has

$$
\begin{aligned}
& \left\langle\widetilde{L} L \phi, I^{m} A^{m-1+\varepsilon} \phi\right\rangle \\
& \quad=\left\langle L \phi,\left(I^{m} \widetilde{L}^{*}+\left[\widetilde{L}^{*}, I^{m}\right]\right) A^{m-1+\varepsilon} \phi\right\rangle \\
& \quad=\left\langle I^{m} L \phi,\left(A^{m-1+\varepsilon} \widetilde{L}^{*}+\left[\widetilde{L}^{*}, A^{m-1+\varepsilon}\right]\right) \phi\right\rangle+\left\langle\left[I^{m}, \widetilde{L}\right] L \phi, A^{m-1+\varepsilon} \phi\right\rangle \\
& \quad \leq C\left(\|L \phi\|_{m}^{2}+\|\widetilde{L} \phi\|_{m-1+\varepsilon}^{2}+\|\phi\|_{m}^{2}\right)
\end{aligned}
$$

and

$$
\begin{aligned}
& \left\langle\widetilde{L} \phi, I^{m} A^{m-1+\varepsilon} \phi\right\rangle \\
& \quad=\left\langle\widetilde{L} \phi,\left(I^{m-1+\varepsilon} L^{*}+\left[L^{*}, I^{m-1+\varepsilon}\right]\right) A^{m} \phi\right\rangle \\
& \quad=\left\langle I^{m-1+\varepsilon} \widetilde{L} \phi,\left(A^{m} L^{*}+\left[L^{*}, A^{m}\right]\right) \phi\right\rangle+\left\langle I^{m-1+\varepsilon} \widetilde{L} \phi, I^{-(m-1+\varepsilon)}\left[L^{*}, I^{m-1+\varepsilon}\right] A^{m} \phi\right\rangle \\
& \quad \leq C\left(\|\widetilde{L} \phi\|_{m-1+\varepsilon}^{2}+\|L \phi\|_{m}^{2}+\|\phi\|_{m}^{2}\right) .
\end{aligned}
$$

Hence,

$$
\|[\widetilde{L}, L] \phi\|_{m-1+\varepsilon / 2}=\left\langle[\tilde{L}, L] \phi,\left.I^{m} A^{m-1+\varepsilon} \phi\right|^{1 / 2} \leq C\left(\|\widetilde{L} \phi\|_{m-1+\varepsilon}+\|L \phi\|_{m}+\|\phi\|_{m}\right) .\right.
$$

Through standard density arguments, one verifies that the above estimate also holds for any $\phi \in H^{m}$ with $\widetilde{L} \phi \in H^{m-1+\varepsilon}$ and $L \phi \in H^{m}$.

Starting from estimate (15) of Theorem 3.3, applying Lemma 4.2 iteratively to elements of $\mathbb{V}_{0}, \ldots, \mathbb{V}_{n_{0}}$, we have the following.

Corollary 4.3. Assume the same hypothesis as in Theorem 3.3. Let condition $(\mathcal{H})$ hold. For the unique solution $(u, v)$ of BSPDE (4), one has further $u \in \mathcal{L}^{2}\left(H^{m+\eta}\right)$ with

$$
E \int_{0}^{T}\|u(t)\|_{m+\eta}^{2} d t \leq C\left\{E\|G\|_{m}^{2}+E \int_{0}^{T}\left(\|f(s)\|_{m}^{2}+\|g(s)\|_{m}^{2}\right) d s\right\},
$$

where the constant $C$ depends on $T, m, n_{0}, \sigma, \theta, b, c$ and $\gamma$.

We are now ready to present the proof of Theorem 4.1 .

Proof of Theorem 4.1. By Theorem 3.3, BSPDE (4) admits a unique solution $(u, v) \in$ $\mathcal{S}^{2}\left(H^{m}\right) \times \mathcal{L}^{2}\left(H^{m-1}\right)$ and the pair of random fields $(\bar{u}, \bar{v})(t, x):=(T-t)(u, v)(t, x)$ turns out to be the unique solution of BSPDE

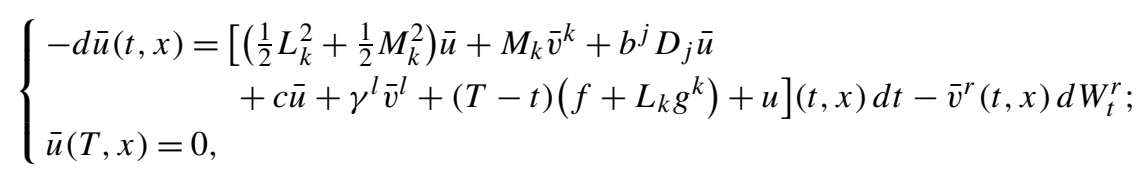


with

$$
\begin{aligned}
& E \sup _{t \in[0, T]}\|\bar{u}(t)\|_{m}^{2}+E \int_{0}^{T}\left(\sum_{k=1}^{d_{1}}\left\|L_{k} \bar{u}(t)\right\|_{m}^{2}+\|\bar{v}(t)+D \bar{u}(t) \theta(t)\|_{m}^{2}\right) d t \\
& \leq C\left(T^{2}+1\right) E \int_{0}^{T}\left(\|f(s)\|_{m}^{2}+\|g(s)\|_{m}^{2}+\|u(s)\|_{m}^{2}\right) d s
\end{aligned}
$$

Starting from the above estimate and applying Lemma 4.2 iteratively to elements of $\mathbb{V}_{0}, \ldots, \mathbb{V}_{n_{0}}$, we have

$$
\begin{aligned}
& \int_{0}^{T}\|D \bar{u}\|_{m-1+\eta}^{2} d s \\
& \quad \leq C\left(T^{2}+1\right) E \int_{0}^{T}\left(\|f(s)\|_{m}^{2}+\|g(s)\|_{m}^{2}+\|u(s)\|_{m}^{2}\right) d s .
\end{aligned}
$$

Fix any $\varepsilon \in(0, T \wedge 1)$ and define $\varepsilon_{l}=\sum_{i=1}^{l} \frac{\varepsilon}{2^{i}}$. By interpolation and Theorem 3.3, one gets

$$
\begin{aligned}
& E \sup _{t \in\left[0, T-\varepsilon_{1}\right]}\|u(t)\|_{m}^{2}+E \int_{0}^{T-\varepsilon_{1}}\left(\|u(t)\|_{m+\eta}^{2}+\|v(t)+D u(t) \theta(t)\|_{m}^{2}\right) d t \\
& \leq \frac{C 2\left(T^{2}+1\right)}{\varepsilon} E \int_{0}^{T}\left(\|f(s)\|_{m}^{2}+\|g(s)\|_{m}^{2}+\|u(s)\|_{m}^{2}\right) d s .
\end{aligned}
$$

Noticing that $(f, g) \in \bigcap_{n \in \mathbb{R}}\left(\mathcal{L}^{2}\left(H^{n}\right) \times \mathcal{L}^{2}\left(\left(H^{n}\right)^{d_{1}}\right)\right)$, by iteration we obtain for any $j \in \mathbb{N}^{+}$,

$$
\begin{aligned}
& E \sup _{t \in\left[0, T-\varepsilon_{j}\right]}\|u(t)\|_{m+(j-1) \eta}^{2}+E \int_{0}^{T-\varepsilon_{j}}\left(\|u(t)\|_{m+j \eta}^{2}+\|v(t)+D u(t) \theta(t)\|_{m+(j-1) \eta}^{2}\right) d t \\
& \leq \frac{C 2^{j}\left(T^{2}+1\right)}{\varepsilon} E \int_{0}^{T-\varepsilon_{j-1}}\left(\|f(s)\|_{m+(j-1) \eta}^{2}+\|g(s)\|_{m+(j-1) \eta}^{2}+\|u(s)\|_{m+(j-1) \eta}^{2}\right) d s
\end{aligned}
$$

which, together with estimate (15), implies by iteration that

$$
\begin{aligned}
& E \sup _{t \in\left[0, T-\varepsilon_{j}\right]}\|u(t)\|_{m+(j-1) \eta}^{2}+E \int_{0}^{T-\varepsilon_{j}}\left(\|u(t)\|_{m+j \eta}^{2}+\|v(t)+D u(t) \theta(t)\|_{m+(j-1) \eta}^{2}\right) d t \\
& \quad \leq C_{j}\left\{E\|G\|_{m}^{2}+E \int_{0}^{T}\left(\|f(s)\|_{m+(j-1) \eta}^{2}+\|g(s)\|_{m+(j-1) \eta}^{2}\right) d s\right\} .
\end{aligned}
$$

Hence, we have

$$
(u, v) \in \bigcap_{n \in \mathbb{R}} L^{2}\left(\Omega ; C\left([0, T-\varepsilon] ; H^{n}\right)\right) \times L^{2}\left(\Omega ; L^{2}\left(0, T-\varepsilon ; H^{n-1}\right)\right) \quad \forall \varepsilon \in(0, T),
$$

and there holds estimate (16). In particular, by Sobolev embedding theorem, the random field $u(t, x)$ is almost surely infinitely differentiable with respect to $x$ and each derivative is a continuous function on $[0, T) \times \mathbb{R}^{d}$. 
At the end of this section, we would show the connection between the conditional expectation (2) and the solution of BSPDE (3).

Proposition 4.4. For the coefficients $G, f, \sigma, \theta, b$, we assume the same hypothesis of Theorem 4.1. Suppose further that $G \in L^{p}\left(\Omega, \mathscr{F}_{T} ; C_{b}\right)$. Let $(u, v) \in \mathcal{S}^{2}\left(H^{m}\right) \times \mathcal{L}^{2}\left(H^{m-1}\right)$ be the solution of BSPDE (3). Then, we have for all $x \in \mathbb{R}^{d}$,

$$
u\left(t, X_{t}^{s, x}\right)=E_{\overline{\mathscr{F}}_{t}}\left[G\left(X_{T}^{s, x}\right)+\int_{t}^{T} f\left(r, X_{r}^{s, x}\right) d r\right] \quad \text { a.s., for all } 0 \leq s \leq t \leq T .
$$

Proof. In view of the continuity of $X_{r}^{s, x}$ with respect to $(s, x, r)$, we first check that all the terms involved in relation (21) make sense in view of the Hörmander-type theorem 4.1. Let $\rho \in C_{c}^{\infty}\left(\mathbb{R}^{d}\right)$ be a nonnegative function with the support in the unit ball centered at the origin such that $\int_{\mathbb{R}^{d}} \rho(y) d y=1$. Define the convolution:

$$
G_{N}(x)=\int_{\mathbb{R}^{d}} G(x-y) \rho(N y) N^{d} d y \quad \text { for } N \in \mathbb{N}^{+} .
$$

In view of the smooth approximation of identity, we have $G_{N} \in \bigcap_{n \in \mathbb{R}} L^{2}\left(\Omega, \mathscr{F}_{T} ; H^{n}\right)$ for each $N \in \mathbb{N}^{+}$, and $G_{N}$ converges to $G$ in space $L^{2}\left(\Omega, \mathscr{F}_{T} ; H^{m}\right)$. Obviously, it holds that $\lim _{N \rightarrow \infty} E\left|G_{N}\left(X_{T}^{s, x}\right)-G\left(X_{T}^{s, x}\right)\right|^{2}=0$ for every $x \in \mathbb{R}^{d}$. For each $N$, let $\left(u_{N}, v_{N}\right)$ be the unique solution of BSPDE (3) with $G$ replaced by $G_{N}$. For each $t \in[0, T)$, by Theorem 4.1, we have for any $\varepsilon \in[0, T-t)$,

$$
\left(u_{N}, v_{N}\right),(u, v) \in \bigcap_{n \in \mathbb{R}} L^{2}\left(\Omega ; C\left([0, t+\varepsilon] ; H^{n}\right)\right) \times L^{2}\left(\Omega ; L^{2}\left(0, t+\varepsilon ; H^{n-1}\right)\right)
$$

and

$$
\left\|u_{N}-u\right\|_{L^{2}\left(\Omega ; C\left([0, t+\varepsilon] ; H^{n}\right)\right)}^{2} \leq C(n) E\left\|G_{N}-G\right\|_{m}^{2} \rightarrow 0 \quad \text { as } N \rightarrow \infty, \forall n \in \mathbb{R},
$$

and in particular, since $H^{d+2}$ is embedded into $C_{b}$, there holds

$$
E \sup _{r \in[s, t+\varepsilon]}\left\|\left(u_{N}-u\right)\left(r, X_{r}^{s, \cdot}\right)\right\|_{\infty}^{2} \leq C\left\|u_{N}-u\right\|_{L^{2}\left(\Omega ; C\left([0, t+\varepsilon] ; H^{d+2}\right)\right)}^{2} \rightarrow 0 \quad \text { as } N \rightarrow \infty .
$$

On the other hand, by the Itô-Kunita formula we have for each $N$ and $0 \leq s \leq t$,

$$
\begin{aligned}
u_{N}\left(t, X_{t}^{s, x}\right)= & G_{N}\left(X_{T}^{s, x}\right)+\int_{t}^{T} f\left(r, X_{r}^{s, x}\right) d r-\int_{t}^{T}\left(v_{N}+D u_{N} \theta\right)\left(r, X_{r}^{s, x}\right) d W_{r} \\
& -\int_{t}^{T} D u_{N} \sigma\left(r, X_{r}^{s, x}\right) d B_{r} \quad \text { a.s., } \forall x \in \mathbb{R}^{d} .
\end{aligned}
$$

Taking conditional expectations on both sides, we get for every $x \in \mathbb{R}^{d}$

$$
u_{N}\left(t, X_{t}^{s, x}\right)=E_{\overline{\mathscr{F}}_{t}}\left[G_{N}\left(X_{T}^{s, x}\right)+\int_{t}^{T} f\left(r, X_{r}^{s, x}\right) d r\right] \quad \text { a.s., for all } 0 \leq s \leq t \leq T .
$$


Letting $N$ go to infinity, we prove (21).

Remark 4.1. In Proposition 4.4 , we assume $G \in L^{2}\left(\Omega, \mathscr{F}_{T} ; C_{b}\right)$ to make sense of the composition $G\left(X_{T}^{t, x}\right)$. We would also remark that by taking $s=t$ in relation (21), the function $u(t, x)$ defined by (2) is just $\mathscr{F}_{t}$-measurable and thus that the conditional expectation in (2) is equivalent to the one with respect to the sub-filtration $\left\{\mathscr{F}_{t}\right\}_{t \geq 0}$, that is,

$$
u(t, x)=E_{\mathscr{F}_{t}}\left[\int_{t}^{T} f\left(r, X_{r}^{t, x}\right) d r+G\left(X_{T}^{t, x}\right)\right], \quad(t, x) \in[0, T] \times \mathbb{R}^{d} .
$$

\section{Acknowledgements}

This paper was finished when the author was in Humboldt-University Berlin, and financial support from the chair Applied Financial Mathematics there is gratefully acknowledged.

\section{References}

[1] Bender, C. and Dokuchaev, N. (2014). A first-order BSPDE for swing option pricing. Math. Finance.

[2] Bensoussan, A. (1983). Maximum principle and dynamic programming approaches of the optimal control of partially observed diffusions. Stochastics 9 169-222. MR0705471

[3] Cass, T. and Friz, P. (2010). Densities for rough differential equations under Hörmander's condition. Ann. of Math. (2) 171 2115-2141. MR2680405

[4] Du, K., Qiu, J. and Tang, S. (2012). $L^{p}$ theory for super-parabolic backward stochastic partial differential equations in the whole space. Appl. Math. Optim. 65 175-219. MR2891221

[5] Du, K., Tang, S. and Zhang, Q. (2013). $\mathrm{W}^{m, p}$-solution $(p \geq 2)$ of linear degenerate backward stochastic partial differential equations in the whole space. J. Differential Equations 254 2877-2904. MR3017034

[6] Graewe, P., Horst, U. and Qiu, J. (2015). A non-Markovian liquidation problem and backward SPDEs with singular terminal conditions. SIAM J. Control Optim. 53 690-711. MR3319844

[7] Hörmander, L. (1967). Hypoelliptic second order differential equations. Acta Math. 119 147-171. MR0222474

[8] Hörmander, L. (1983). The Analysis of Linear Partial Differential Operators. II. Grundlehren der Mathematischen Wissenschaften 257. Berlin: Springer. MR0705278

[9] Hu, Y., Ma, J. and Yong, J. (2002). On semi-linear degenerate backward stochastic partial differential equations. Probab. Theory Related Fields 123 381-411. MR1918539

[10] Hu, Y. and Peng, S.G. (1991). Adapted solution of a backward semilinear stochastic evolution equation. Stoch. Anal. Appl. 9 445-459. MR1133858

[11] Krylov, N.V. (2013). Hörmander's theorem for stochastic partial differential equations. Available at arXiv: 1309.5543.

[12] Krylov, N.V. and Rozovskii, B.L. (1981). Stochastic evolution equations. J. Sov. Math. 16 1233-1277.

[13] Ma, J. and Yong, J. (1999). On linear, degenerate backward stochastic partial differential equations. Probab. Theory Related Fields 113 135-170. MR1676768

[14] Malliavin, P. (1978). Stochastic calculus of variation and hypoelliptic operators. In Proceedings of the International Symposium on Stochastic Differential Equations (Res. Inst. Math. Sci., Kyoto Univ., Kyoto, 1976) 195-263. New York: Wiley. MR0536013 
[15] Mattingly, J.C. and Pardoux, É. (2006). Malliavin calculus for the stochastic 2D Navier-Stokes equation. Comm. Pure Appl. Math. 59 1742-1790. MR2257860

[16] Peng, S.G. (1992). Stochastic Hamilton-Jacobi-Bellman equations. SIAM J. Control Optim. 30284 304. MR1149069

[17] Qiu, J. and Tang, S. (2012). Maximum principle for quasi-linear backward stochastic partial differential equations. J. Funct. Anal. 262 2436-2480. MR2876411

[18] Qiu, J. and Wei, W. (2014). On the quasi-linear reflected backward stochastic partial differential equations. J. Funct. Anal. 267 3598-3656. MR3266242

[19] Stroock, D.W. and Varadhan, S.R.S. (1979). Multidimensional Diffusion Processes. Grundlehren der Mathematischen Wissenschaften 233. New York: Springer. MR0532498

[20] Tang, S. and Wei, W. (2016). On the Cauchy problem for backward stochastic partial differential equations in Hölder spaces. Ann. Probab. 44 360-398. MR3456341

[21] Zhou, X.Y. (1992). A duality analysis on stochastic partial differential equations. J. Funct. Anal. 103 275-293. MR1151549

Received March 2015 and revised January 2016 